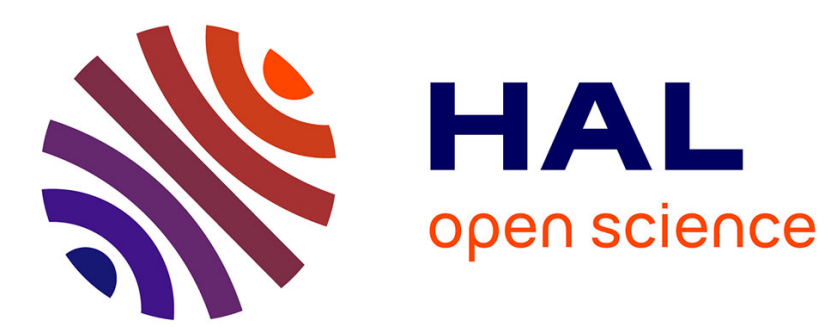

\title{
Acoustically bound microfluidic bubble crystals
}

David Rabaud, Pierre Thibault, Mylène Mathieu, Philippe Marmottant

\section{To cite this version:}

David Rabaud, Pierre Thibault, Mylène Mathieu, Philippe Marmottant. Acoustically bound microfluidic bubble crystals. Physical Review Letters, 2011, 106, pp.134501. 10.1103/PhysRevLett.106.134501 . hal-00582406

\section{HAL Id: hal-00582406 \\ https://hal.science/hal-00582406}

Submitted on 1 Apr 2011

HAL is a multi-disciplinary open access archive for the deposit and dissemination of scientific research documents, whether they are published or not. The documents may come from teaching and research institutions in France or abroad, or from public or private research centers.
L'archive ouverte pluridisciplinaire HAL, est destinée au dépôt et à la diffusion de documents scientifiques de niveau recherche, publiés ou non, émanant des établissements d'enseignement et de recherche français ou étrangers, des laboratoires publics ou privés. 


\title{
Acoustically Bound Microfluidic Bubble Crystals
}

\author{
David Rabaud, Pierre Thibault, Mylène Mathieu, and Philippe Marmottant* \\ CNRS \& Université de Grenoble, UMR 5588, Laboratoire Interdisciplinaire de Physique, Grenoble, F-38041, France
}

(Received 13 December 2010; published 29 March 2011)

\begin{abstract}
Bubbles confined in microchannels self-organize without directly contacting one another when excited by an external acoustic field. The bubbles tend to form periodic "crystal"-like lattices with a finite interbubble distance. This equilibrium distance can be adjusted by simply tuning the acoustic frequency. This new type of crystal is purely mediated by acoustic surface waves emitted by the pulsating bubbles. Because these waves are reflected at the channel boundaries, the bubbles interact with their own images across the boundary.
\end{abstract}

DOI: 10.1103/PhysRevLett.106.134501

PACS numbers: 47.55.db, 47.60.-i, 83.50.Ha, 83.80.Iz

Introduction.-Although acoustic waves are well known as convenient tools to passively probe materials, they can also lead to acoustic forces that can actively translate objects embedded in a fluid medium. Compared to other objects such as solid particles or droplets, a bubble in a liquid has a strong vibrational response to acoustic waves because its gas content is much more compressible. Since its volume pulsates significantly [1], a bubble efficiently scatters ultrasound waves and becomes a secondary sound emitter. When undergoing this oscillation, the bubble is subjected to a strong force from incoming acoustic waves called the primary acoustic Bjerknes force [2]. The bubble may also experience a secondary Bjerknes force from neighboring bubbles. These forces are at play in numerous acoustic applications, such as in medical ultrasonics, acoustic degassing, multibubble cavitation and sonoluminescence [1].

Bubbles of the same size experience an attractive interaction at long distances, as observed and predicted by a simple model $[3,4]$. However, a singular observation showed that they agglomerate but do not necessarily coalesce: these "bubble grapes" were first described by [5]. This report (confirmed by [6,7]) suggested that bubbles could be maintained without contact at a small, finite separation distance, even if no value was measured. This finding in turn triggered many theoretical approaches that predicted a repulsive interaction at short distances and, therefore, a finite equilibrium distance $[1,8-13]$. These hypotheses have not been confirmed by experiments.

Here, we show that the interaction between bubbles can indeed present a marked short-range repulsion and therefore a finite equilibrium distance when bubbles are confined in microchannels: bubbles self-organize into "acoustically bound crystals" with purely acoustic interactions. This interaction is dominant over hydrodynamic effects [14].

The physical system we introduce appears as an interesting model system in the context of complex systems. Bubbles are simple objects and are coupled to any incident external field from which they can obtain mechanical energy. As a secondary effect, bubbles are acoustically coupled to their neighbors, which provides the basis of the interactions in this multiagent system.

Results.-We designed a microfluidic planar setup for the purpose of studying microscopic bubbles (Fig. 1). The bubbles, $20-50$ micrometers in radius, were generated by a flow-focusing geometry [15], which facilitates the production of bubbles with extremely reproducible sizes $[15,16]$ that depend only on the applied gas pressure and the liquid flow rate. A surfactant was added to the liquid to prevent bubble coalescence. The bubbles were confined in channels made entirely of a polydimethylsiloxane (PDMS) elastomer using standard soft lithography techniques [17]. The channels were shallow ( $h=25 \mu \mathrm{m}$ in depth), and the bubbles were therefore in contact with the transparent top and bottom surfaces; this contact was lubricated by a thin liquid film. An acoustic field was applied locally through an intermediate- a rectangular glass rod that was molded in the elastomer just above the channel (145 $\mu \mathrm{m}$ away). The rod was vibrated by a piezoelectric element glued onto it. The channel therefore received the ultrasound field emitted by the rod through the elastomer, which transmitted the vibration.

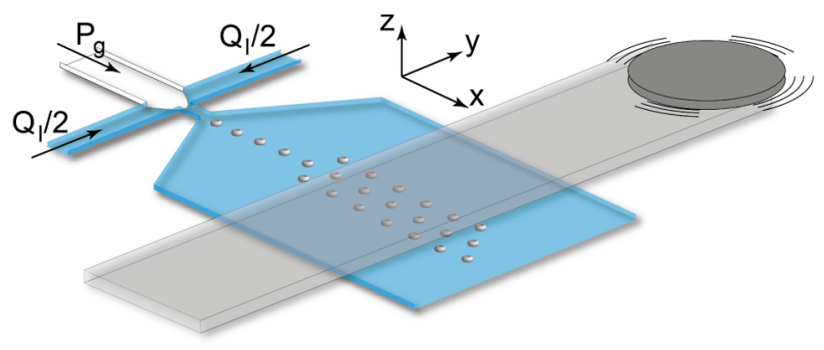

FIG. 1 (color online). The microfluidic setup. The liquid is sketched in blue (dark gray), the gas in white and the vibrating glass rod in light gray (molded above the channel). Bubbles are produced in the flow-focusing section and then flow into a large channel where sound is applied locally via the vibration of the rod. 


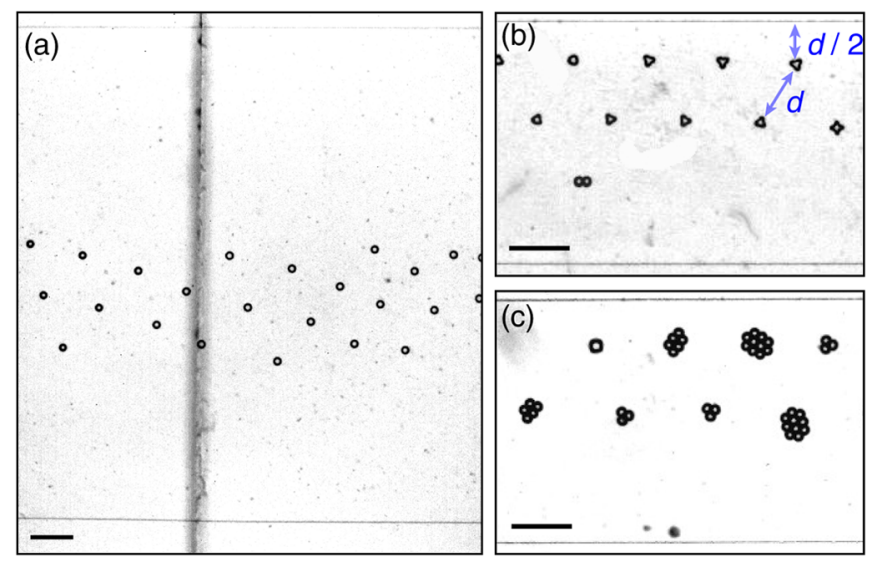

FIG. 2 (color online). Experimental snapshots showing acoustically bound flowing crystals, with bubbles whose radii are around $25 \mu \mathrm{m}$. (a) Periodic triangular arrays after self-organization. The equilibrium distance is $340 \mu \mathrm{m}$ ( $f=87 \mathrm{kHz}$ ). (b) Effect of the channel boundaries (thin horizontal lines): the bubble tend to be attracted at a distance that is half the crystal distance $d$ ( $f=126 \mathrm{kHz}$ ). (c) Bubbles can form aggregates at nodes of the periodic array $(f=90 \mathrm{kHz})$. In all of the pictures, the area under the rectangular glass rod is located to the right of the vertical line. The bubbles flow from left to right. The scale bar is $250 \mu \mathrm{m}$ in all images.

For a sufficient acoustic amplitude, we observed that the bubbles, which were initially in a line after the flowfocusing generator, spontaneously self-organized in a balletlike motion while keeping an equilibrium distance. The bubbles finally arranged themselves into regular hexagonal patterns, and an "acoustically bound crystal" was created (Fig. 2). This crystal was not stationary but was advected by the flow (movie 1 in Ref. [18]). The bubble positions were therefore not imposed by the standing external field (as in a Kundt tube or a resonant cavity [19]) but instead plainly resulted from their mutual interactions.

This crystal was tunable: the distance between the bubbles could be adjusted by switching the acoustic frequency (Fig. 3). Surprisingly, this distance did not depend on the bubble size or on the acoustic amplitude (insert in Fig. 3).

For large initial bubble densities, aggregates of several contacting bubbles were seen [Fig. 2(c)]. These aggregates also arranged themselves into a crystalline order with an equilibrium distance that is comparable to crystals made of single bubbles.

At large acoustic amplitudes, the bubble surface displayed undulations [Fig. 2(b)]. A parametric instability, the Faraday instability [20], occurred, which led to standing waves on each bubble's surface. We observed between three and ten wavelengths of undulation around the perimeter, depending on the bubble's apparent diameter. These values are close to the predictions by Lamb [21-23] for spherical bubbles (movies 2-4 and Fig. 1 in Ref. [18]). Nonetheless, the appearance of these "gearlike" bubbles did not affect the crystal organization.

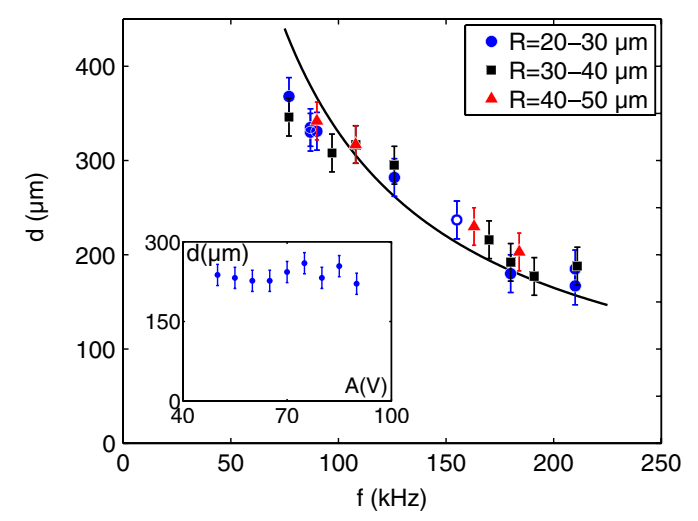

FIG. 3 (color online). The interbubble distance and its variation with the frequency and the excitation voltage of the ultrasound transducer (insert) for different bubble sizes. The line is a fit to $d=c / f$, with $c=33 \mathrm{~m} / \mathrm{s}$. Error bars represent the standard deviation over several measurements.

Model.-Despite the lack of experimental proof available, intense theoretical efforts that have predicted short-range repulsion forces can be found in the literature. However, none of these studies predict an equilibrium distance that is independent of the bubble radius and the oscillation amplitude. These models can be classified under four different types of assumptions. (i) The bubbles act as coupled oscillators at small distances [8]. This model predicts a decrease in the resonance frequency when two bubbles get close to each other, and it identifies a repulsion force at short distances, but only for bubbles of different sizes. (ii) Interference arises between the acoustic pressures emitted by the bubbles and the primary sound field $[1,9]$. This theory predicts an equilibrium at small distances that strongly depends on the bubble size (which determines the phase of the bubble oscillation). (iii) The bubble oscillation is nonlinear [10-12]. A repulsive interaction only occurs at very large pulsation amplitudes. (iv) The bubbles emit acoustic waves that propagate with a finite wavelength in the bulk [13]. This model predicts an equilibrium distance on the order of the wavelength of sound, which is much larger than the one we observed. In addition, acoustic streaming forces are not expected to play a role because of the spherical pulsation of bubbles, without any translation (contrary to particles in [24]).

To interpret our observations, we derived a new model based on the fact that vibrating bubbles also emit surface acoustic waves in addition to bulk waves. In our setup, the channel walls were made of soft elastomeric PDMS so that the bubble vibration could drive two-dimensional surface Rayleigh waves [25] [Fig. 4(a)]. These secondary waves propagate from each bubble. We started by assuming that all bubble volumes pulsate in phase according to $V=V_{0}[1+\varepsilon \cos (\omega t)]$, where $\varepsilon$ is the relative amplitude of vibration and $\omega=2 \pi f$. The pressure in the liquid due 
(a)
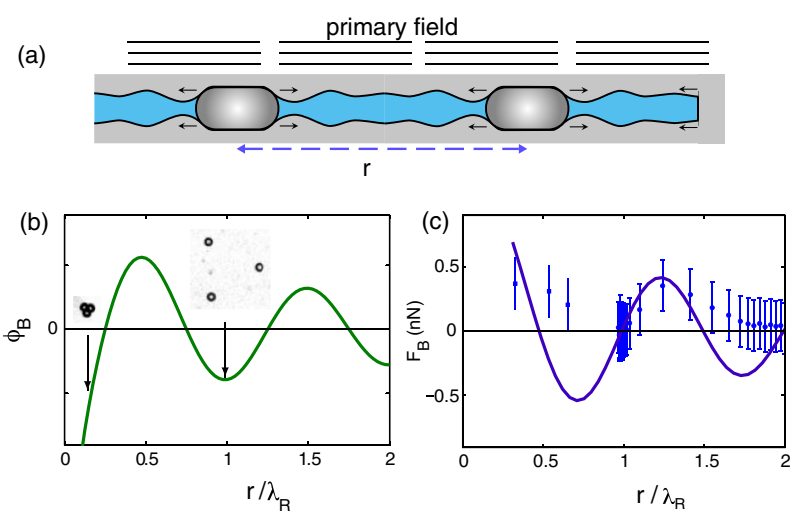

FIG. 4 (color online). (a) A cartoon showing the secondary emission of surface waves, as seen from the side. The PDMS walls are in grey, while the liquid-filled channel is in blue (dark grey). The surface waves reflect on the side boundaries, here on the right boundary. Surfaces waves are of the Rayleigh type when $\lambda_{R}$ is much smaller than the wall thickness [25], a condition that is always fulfilled for the millimeter-thick bottom wall, and only at high frequency for the $145 \mu$ m-thick top wall. (b) The acoustic interaction potential from Eq. (1). (c) The force deriving from this potential (line) and measured from the approach velocity between two bubbles: circles represent crystal assembly, and squares represent aggregation. Same conditions at $f=155 \mathrm{kHz}$. Error bars represent uncertainty. The parameters for the predicted interaction are $\lambda=226 \mu \mathrm{m}$ and vibration amplitude $\varepsilon=0.059$.

to the surface wave in the fluid is $P_{2}=-P_{2,0}\left(R_{0} / r\right)^{1 / 2}$ $\cos \left(\omega t-k_{R} r\right)$, where $r$ is the distance from the bubble's center, $R_{0}$ is the bubble's radius at rest, $k_{R}=2 \pi / \lambda_{R}$ is the Rayleigh wave number, and $P_{2,0}$ is the liquid pressure emitted by the bubble. The order of magnitude for the pressure in the liquid is easy to estimate [26]. Because the surface wave velocity in PDMS is much smaller than the sound velocity in the liquid (as we will discuss below), there is no sound emission in the liquid, but only an evanescent pressure field above the surface on a layer of typical thickness $\lambda_{R}$ [25,27]. This original situation contrasts with that of hard solid materials such as silicon, which has a surface wave velocity higher than the liquid, leading to radiative losses or "leaky waves." Note that more rigid solid polymers (such as PMMA), also present a surface wave velocity lower than sound, but to a lesser extent [28]. Here, we do not take into account the interaction between the top and bottom surface vibrations, and assume that the evanescent pressure in the liquid is homogeneous in the channel gap (since its height is small compared to the wavelength). The secondary acoustic Bjerknes force is simply the time average of the evanescent pressure forces on a neighboring bubble at a distance $r: \mathbf{F}_{B}=\left\langle-V(t) \nabla P_{2}(t, r)\right\rangle$. Using the expressions for the volume and the pressure, we find that the acoustic Bjerknes force is aligned with the bubble centers, and its amplitude derives from a potential:
$F_{B}(r)=-\frac{\partial \Phi_{B}}{\partial r} \quad$ with $\quad \Phi_{B}=-\frac{1}{2} \varepsilon V_{0} P_{2,0} \sqrt{\frac{R_{0}}{r}} \cos \left(k_{R} r\right)$.

This potential is plotted in Fig. 4(b), while the force is plotted in Fig. 4(c). The interaction is attractive at small distances, as reported in the literature. This interaction predicts agglomerated bubble clusters. However, the potential oscillates with the distance, which remarkably produces equilibrium positions at finite distances. The first minimum occurs at one wavelength $r=\lambda_{R}$, which accounts for the distance that is experimentally observed. There are secondary minima at distances that are multiples of $\lambda_{R}$, but they are less remarkable because they are situated in shallower wells of the potential. Following this model, the wavelength, and consequently the equilibrium distance, depend only on the frequency through the relationship $d=\lambda_{R}=c_{R} / f$, where $c_{R}$ is the velocity of the surface waves.

Discussion.-The observed equilibrium distances are qualitatively and quantitatively compatible with the wavelength just introduced. The measurements are described by $d \simeq c / f$, with $c=33 \mathrm{~m} / \mathrm{s}$. This velocity for surface waves, which is much smaller than the velocity of sound in liquid (around $1500 \mathrm{~m} / \mathrm{s}$ ), is in good agreement with the material stiffness of the walls. A velocity of $c_{R}=33 \mathrm{~m} / \mathrm{s}$ was computed for a Young's modulus of 3.3 MPa [29], which is well within the range of static values measured for PDMS, namely, 0.8-4 MPa [30], depending on the age and curing time of the elastomer.

One may also wonder if 3D crystals could be observed in bulk water. We believe this would be difficult because the Bjerknes interaction would be much weaker. The propagating pressure emitted by a pulsating bubble decays as $r^{-1}$ in 3D, which is faster than the $r^{-1 / 2}$ decay in 2D. The wavelength of bulk waves is also much larger (about 50 times). The project would therefore require extremely high amplitude oscillations - so high, in fact, that the stability of the bubble would no longer be warranted.

Moreover, the shape of the potential itself can be retrieved through force measurements. Because of the negligible inertia in the translational motion of bubbles at these tiny scales, friction forces exactly balance the force acting on the bubbles. The friction force is inferred from the measured velocity vector: The friction force of a confined bubble of velocity $\mathbf{v}$ is mainly due to friction with the walls and may be expressed as $\mathbf{F}_{f}=\alpha(v) \mu h \mathbf{v}$, with $\mu$ the fluid viscosity. The friction coefficient $\alpha$ decreases with velocity because faster bubbles are lubricated by thicker films. We calibrated $\alpha(v) \simeq 11(h / R)^{3 / 2}(\mu v / \sigma)^{-1 / 2}$ in the presence of surfactant [31], with $\sigma$ the surface tension of fluid $(\simeq 30 \mathrm{mN} / \mathrm{m})$. The acting forces on a bubble's trajectory during assembly or during aggregation are displayed in Fig. 4(c). In this particular experiment, we found 
from Eq. (1) and from the estimation of $P_{2,0}$ that the vibration amplitude is indeed small: $\varepsilon=5.9 \times 10^{-2}$.

The model of surface waves is further supported by the fact that the bubbles also interacted with the side boundaries of the channel. It was observed that they kept a distance from the boundary that was equal to about half the distance between the bubbles [Fig. 2(b)], or the bubbles squarely contact the wall. This interaction can be explained by the fact that surface waves are reflected by the boundaries, and after reflection are equivalent to those emitted by "image" bubbles, placed symmetrically on the other side of the boundary. Bubbles therefore interact with their images, which produces this "Narcissus" effect [32].

Conclusion.-The self-organization we report, based on a wave-particle duality, shares common features with the bouncing of liquid drops at the surface of a vibrating liquid [33]. However, there are two main qualitative differences: the first is that the bubble interaction does not use gravity, and the second is that the excitation amplitude is small, with no clear critical amplitude.

Our understanding of bubble organization allows us to envision the fabrication of bubble arrangements at a micrometric size. The generation of "crystal on demand" opens promising perspectives for creating adaptive phononic arrays, tuned by the excitation frequency. In the same spirit, the control of periodically arranged voids would be interesting as templates to synthesize new materials.

*philippe.marmottant@ujf-grenoble.fr

[1] T. G. Leighton, The Acoustic Bubble (Academic Press, London, 1994).

[2] V.F. J. Bjerknes, Fields of Force (Columbia University Press, New York, 1906).

[3] L. A. Crum, J. Acoust. Soc. Am. 57, 1363 (1975).

[4] V. Garbin, B. Dollet, M. Overvelde, D. Cojoc, E. Di Fabrizio, L. van Wijngaarden, A. Prosperetti, N. de Jong, D. Lohse, and M. Versluis, Phys. Fluids 21, 092003 (2009).

[5] Y. A. Kobelev, L. A. Ostrovskii, and A. M. Sutin, JETP Lett. 30, 395 (1979).

[6] P.L. Marston, E. H. Trinh, J. Depew, and T. J. Asaki, in Bubble Dynamics and Interface Phenomena, edited by J.R. Blake, J.L. Boulton-Stone, and N.H. Thomas (Kluwer, Dordrecht, The Netherlands, 1994).

[7] A. A. Doinikov and S. T. Zavtrak, J. Acoust. Soc. Am. 99, 3849 (1996).

[8] E. A. Zabolotskaya, Sov. Phys. Acoust. 30, 365 (1984).

[9] A. A. Doinikov and S. T. Zavtrak, Phys. Fluids 7, 1923 (1995).

[10] H. N. Oguz and A. Prosperetti, J. Fluid Mech. 218, 143 (1990).

[11] R. Mettin, I. Akhatov, U. Parlitz, C. D. Ohl, and W. Lauterborn, Phys. Rev. E 56, 2924 (1997).
[12] A. A. Doinikov, Phys. Rev. E 64, 026301 (2001).

[13] A. A. Doinikov, J. Fluid Mech. 444, 1 (2001).

[14] T. Beatus, T. Tluskty, and R. Bar-Ziv, Nature Phys. 2, 743 (2006).

[15] P. Garstecki, I. Gitlin, W. DiLuzio, G. M. Whitesides, E. Kumacheva, and H. A. Stone, Appl. Phys. Lett. 85, 2649 (2004).

[16] B. Dollet, W. van Hoeve, J.-P. Raven, P. Marmottant, and M. Versluis, Phys. Rev. Lett. 100, 034504 (2008).

[17] D. Duffy, J. McDonald, O. Schueller, and G. Whitesides, Anal. Chem. 70, 4974 (1998).

[18] See supplemental material at http://link.aps.org/ supplemental/10.1103/PhysRevLett.106.134501 for movies showing the evolution of the crystal and the bubble undulations, together with a figure displaying the number of surface undulations.

[19] J. Shi, D. Ahmed, X. Mao, S.-C.S. Lin, A. Lawit, and T. J. Huang, Lab Chip 9, 2890 (2009).

[20] S. Chandrasekhar, Hydrodynamic And Hydromagnetic Stability (Dover publication, New York, 1961).

[21] H. Lamb, Hydrodynamics (Cambridge University Press, Cambridge, 1932).

[22] M. Versluis, D. E. Goertz, P. Palanchon, I. L. Heitman, S. M. van der Meer, B. Dollet, N. de Jong, and D. Lohse, Phys. Rev. E 82, 026321 (2010).

[23] R. Dangla and C. Poulain, Phys. Fluids 22, 041703 (2010).

[24] G. A. Voth, B. Bigger, M. R. Buckley, W. Losert, M. P. Brenner, H. A. Stone, and J. P. Gollub, Phys. Rev. Lett. 88, 234301 (2002).

[25] I. A. Viktorov, Rayleigh and Lamb Waves (Plenum Press, New York, 1967).

[26] We can estimate the pressure in the vicinity of the pulsating bubble by writing the Euler relation for a fluid of density $\rho_{f}$ at the bubble's surface: $\rho_{f} d^{2} R / d t^{2}=$ $-\partial_{r} P_{2}(r=R)$. With the assumption of a cylindrical vibration of the quasi-2D bubbles such that $V(t)=\pi R(t)^{2} h$, we obtain $P_{2,0}=\varepsilon \rho_{f} \omega^{2} R_{0}^{2}$ (when $\left.R_{0} \ll \lambda_{R}\right)$.

[27] D. Royer and E. Dieuleusaint, Elastic Waves In Solids I: Free And Guided Propagation (Springer, Berlin, 2000).

[28] B. T. Hefner and P. L. Marston, J. Acoust. Soc. Am. 107, 1930 (2000).

[29] In the case of polymeric materials the Poisson's ratio is equal to $\nu=1 / 2$, and $c_{R}$ is close to the velocity $c_{S}$ of bulk shear waves in the solid, i.e., $c_{R} \simeq 0.96 c_{S}$ [25]. The shear wave velocity writes $c_{S}=\left\{E /\left[2(1+\nu) \rho_{s}\right]\right\}^{1 / 2}$, with the Young's modulus $E$, and the density $\rho_{S}$ of the solid.

[30] D. Fuard, T. Tzvetkova-Chevolleau, S. Decossas, P. Tracqui, and P. Schiavone, Microelectron. Eng. 85, 1289 (2008).

[31] D. Rabaud, P. Thibault, J.-P. Raven, O. Hugon, E. Lacot, and P. Marmottant, hal.archives-ouvertes.fr:hal-00576528 [Phys. Fluids (to be published)]..

[32] P. Marmottant, M. Versluis, N. de Jong, S. Hilgenfeldt, and D. Lohse, Exp. Fluids 41, 147 (2006).

[33] S. Protière, A. Boudaoud, and Y. Couder, J. Fluid Mech. 554, 85 (2006). 